\title{
Refinery: Visual Exploration of Large, Heterogeneous Networks through Associative Browsing
}

\author{
S. Kairam ${ }^{1}$, N. H. Riche ${ }^{2}$, S. Drucker ${ }^{2}$, R. Fernandez ${ }^{2}$, and J. Heer ${ }^{3}$ \\ ${ }^{1}$ Computer Science Department, Stanford University \\ ${ }^{2}$ Microsoft Research \\ ${ }^{3}$ Computer Science \& Engineering, University of Washington
}

\begin{abstract}
Browsing is a fundamental aspect of exploratory information-seeking. Associative browsing encompasses a common and intuitive set of exploratory strategies in which users step iteratively from familiar to novel pieces of information. In this paper, we consider associative browsing as a strategy for bottom-up exploration of large, heterogeneous networks. We present Refinery, an interactive visualization system informed by guidelines drawn from examination of several areas of literature related to exploratory information-seeking. These guidelines motivate Refinery's query model, which allows users to simply and expressively construct queries using heterogeneous sets of nodes. The system ranks and returns associated content using a fast, random-walk based algorithm, visualizing results and connections among them to provide explanatory context, facilitate serendipitous discovery, and stimulate continued exploration. A study of 12 academic researchers using Refinery to browse publication data related to areas of study demonstrates how the system complements existing tools in supporting discovery.
\end{abstract}

Categories and Subject Descriptors (according to ACM CCS): H.5.2 [Information Interfaces and Presentation]: User Interfaces-H.3.3 [Information Storage and Retrieval]: Information Search and Retrieval—

\section{Introduction}

Navigating electronic collections often requires a variety of strategies, which have been classified broadly into two categories, analytical and browsing [MS88]. While analytical strategies are used for retrieving specific facts, browsing is used more for exploratory information-seeking tasks, defined here as learning about and investigating a knowledge domain via continuous, iterative interaction with available resources [Be193, Mar06, WKDS06].

Prior research in HCI has found that searchers engaged in exploration often naturally adopt strategies based on orienteering [OJ93, TAAK04], navigating towards information goals using small, iterative steps using cues from the environment. As the term orienteering has been used recently to describe navigating towards known information targets [TAAK04, CMF08], we use the more general term associative browsing to refer to cases in which the information goal is either a specific topic or general knowledgegathering. We contrast these strategies with teleporting, or using keyword search and other means to navigate immediately to desired pieces of content. In cases where tele- porting is impossible or unwieldy, associative browsing offers benefits such as circumventing difficulties in specifying queries [FLGD87, tHPvdW96, TAAK04] and providing explanatory context for results [TAAK04, DCW11].

In this paper, we present Refinery, a visualization system for exploring large, heterogeneous networks through associative browsing. Despite the varied benefits of associative strategies for exploration, there has been limited work examining how to design visualization system specifically to support them, especially in the area of network visualization.

We first review literature on exploratory informationseeking, identify guidelines for designing interfaces to support associative browsing, and examine techniques used by several classes of existing systems for instantiating these guidelines. We then describe the design and implementation of Refinery. Finally, we present the results of a study of 12 academic researchers using the system to browse conference publication data. We observe how they use Refinery to explore new research areas and discover novel insights within their existing areas of expertise. 
The primary contributions of this work are as follows:

- We identify design guidelines and strategies for interactive visualization systems to support associative browsing.

- We present Refinery, a system which uniquely instantiates these strategies to enable effective bottom-up visual exploration of heterogeneous networks.

- We describe a novel application of random-walk based graph algorithms to the problem of extending degree-ofinterest (DOI) visualization to heterogeneous networks.

\section{Developing Design Goals for Associative Browsing}

In this section, we review prior work from several areas relevant to exploratory information-seeking in order to develop a set of high-level guidelines for designing visual interfaces for associative browsing over complex data.

\subsection{Background: Exploratory Information-Seeking}

For decades, information retrieval focused on matching user queries to documents. As discussed by Marchionini \& Shneiderman [MS88], the advent of hypertext collections offered diverse possibilities for navigation, with analytical retrieval tasks complemented by browsing, which is more continuous and iterative in nature. Using the metaphor of berrypicking, Bates [Bat89] observed that individuals pick up bits and pieces of information as they navigate through an information space. Belkin [Be193] outlines how searchers not only accumulate knowledge but also change their perception of the search task through interaction with information in the environment.

Studying individuals searching library collections, O'Day \& Jeffries [OJ93] observed how searchers examined results returned by librarians and used these to guide future search iterations. Teevan et al. [TAAK04] observed similar strategies for users browsing electronic information on their personal computers. Despite the different contexts and goals, searchers in both studies naturally adopted an iterative process of leveraging contextual cues to choose subsequent exploration steps until search goals were achieved.

One observed benefit of adopting associative strategies is in circumventing difficulties in composing queries. The well-known "vocabulary problem" [FLGD87] stems from the fact that searchers often have to choose from many possible search terms for a given target. In addition, difficulty recalling details about the target early in the search often makes keyword search untenable [TAAK04]. For searchers without a clear notion of the target, Bruza [Bru93] observes that they can usually spot relevant information when it appears, offering the quote "I don't know what I'm looking for, but I'll know it when I find it." ter Hofstede [tHPvdW96] identifies an interactive query formulation loop with three phases - exploration, construction, and feedback — which searchers repeat until achieving the information goal. The

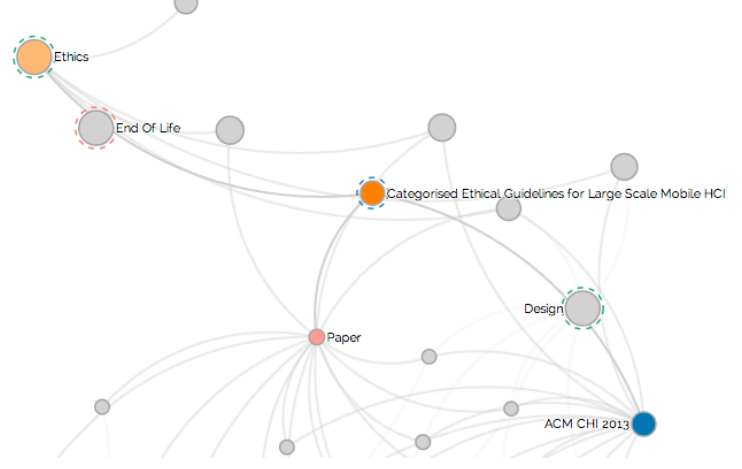

Figure 1: Refinery allows users to explore large, heterogeneous networks by following connections from known items to associated content. The visualized connections provide both explanatory context and exploratory possibilities.

Mr. Taggy system [KNPC09] illustrates how tightening this loop through active suggestion of query refinements can facilitate exploration and sensemaking.

In the case of browsing for known information targets, these observations relate to psychological theories holding that memory is encoded and retrieved in the form of interitem associations. Anderson \& Pirolli [AP84] describe how observed information triggers associations to content in a long-term memory through a process of spreading activation. Earlier experimental work by Tulving [TT73] suggests that retrieval of items from memory can be improved by providing particular associations which were present when the information was initially encoded.

In the case of more general exploration, where searchers aim to uncover previously unknown items, we look to literature on serendipitous finding. Andre et al. [ASTD09] characterize serendipity as the combination of finding unexpected information and the ability to make an intellectual leap to connect that information to what you already know. Marchionini \& Shneiderman [MS88] consider such serendipitous finding to be central to the activity of browsing. Dörk et al. [DCW11] advise that serendipity can be encouraged in visualization interfaces by "juxtaposing resources that share unusual facets or relate to one's previous interactions."

\subsection{Guidelines for Supporting Associative Browsing}

Based on these findings, as well as challenges observed in real-world exploratory tasks, we offer the following guidelines for designing interactive visualization systems to support associative browsing over complex information spaces.

G1. Support browsing across heterogeneous, dynamic collections. Hypertext information collections encompass a variety of media types, and an associative browser should enable exploration across both textual and non-textual data, such as images or videos [MS88, Mar04]. As data are fre- 
S. Kairam et al. / Refinery: Visual Exploration of Large, Heterogeneous Networks through Associative Browsing

quently combined from disparate sources with perhaps different schemas, it is important that we build a common language model for flexibly representing pieces of content and their relationships [ASTD09]. Computation driving our application should be done on the fly, in order to accommodate the increasingly dynamic nature of available information resources (e.g. news, blogs, social media) [Mar04].

G2. Balance simplicity and expressivity in representing search intent. In information retrieval settings, keywordbased search can be highly expressive, but the large set of possible choices can lead to roadblocks [FLGD87, Bru93,tHPvdW96]. However, the system should be sufficiently expressive to allow users to choose queries specific enough to focus exploration in areas of interest. Once users have begun exploring, the system should suggest and allow users to easily evaluate possible query refinements [TAAK04].

G3. Refine search intent through continuous dialog with the user. The interface should aim to engage users directly and actively in the information retrieval process [Be193], favoring a continuous interactive dialog over more formalized turn-taking [Mar06]. To allow the dialog to continue smoothly, the system should make it easy to provide relevance feedback about suggested items [Mar06, KNPC09].

G4. Surface varied contextual cues to support recognition and discovery. The observation that recognition of content improves when it is presented with context matching that in which it was encoded [TT73] suggests the need for offering a diverse set of contextual cues and connections for returned items. These cues can facilitate recognition of target items when uncovered [Bru93, TAAK04] or serendipitous finding of useful, novel items [ASTD09, DCW11].

\subsection{Related Systems}

In this section, we look to several classes of existing systems related to exploratory information-seeking. For each class, we highlight one system as an example to illustrate techniques for instantiating these guidelines. These observations are summarized in Table 1.

Faceted and Cluster-Based Browsing. This class of interfaces allow users to leverage item metadata to iteratively explore collections. Faceted browsing allows users to specify filters using metadata to find subsets of items sharing specific desired characteristics. A study of the Flamenco system [YSLH03] illustrated how metadata could help users browse large image collections more easily than keyword search alone. Clustering achieves a similar goal by using metadata to group items with similar properties. Studying category usage in the Findex system, Käki et al. [K0̈5] observed how clusters helped users to refine queries when initial keyword searches failed. Rodden, et al. [RBSW01] observed the important role that decisions about strategies for categorizing images play in subsequent browsing behavior.

We highlight Flamenco as a well-known example of these

\begin{tabular}{|c|c|c|c|c|}
\hline & G1 & G2 & G3 & G4 \\
\hline \hline Faceted/Clustered Browers & - & + & + & - \\
\hline DOI Visualization & - & o & + & + \\
\hline Ostensive Browsers & - & + & + & o \\
\hline
\end{tabular}

Table 1: Summary of extent to which stated guidelines are supported by several classes of existing systems.

techniques. Flamenco combines keyword search with easily selectable facets, providing multiple means of specifying search intent (G2). The system enables users to iteratively select facets and receive suggestions for refinements to navigate easily towards search goals (G3). Flamenco and related systems (e.g. FacetLens [?] \& PivotSlice [?]), however, only enable browsing items of a single type and do not allow for non-textual queries (e.g. using images, video, etc.) ( $\neg$ G1). Furthermore, both faceting and clustering hide relationships among items within a visible group or across groups $(\neg \mathbf{G 4})$, possibly hiding opportunities for exploration and discovery. One exception here is the PivotSlice system, which displays relationships among items of the same type.

Degree-of-Interest Visualization. Degree-of-interest (DOI) visualization techniques, as proposed by Furnas [Fur86], highlight or magnify items of interest along with a subset of items which may provide explanatory context. Techniques which increase the visual saliency of important neighbors have been applied to tree [CN02, HC04] and graph [LPP*] structures. van Ham \& Perer [vHP09] proposed a network exploration system enabling DOI scoring of non-neighbor nodes through a function combining three elements: a priori interest (API), user interest (UI) based on the user's query, and distance from nodes in focus.

Using van Ham \& Perer's system as an example, we see that DOI systems are built around the notion of visualizing contextually relevant information for a particular object of interest (G4). The interactive system they describe allows users to select these contextually relevant items and add them to the current focus, facilitating rapid refinement of the view (G3). It is non-trivial, however, to extend their formulation to heterogeneous networks $(\neg \mathbf{G 1})$. One problem is defining separate UI functions for each type of node. Learning to rank documents against images and other types of content for each new dataset would require significant research and refinement. In addition, their approach is openended with respect to how queries are specified (? G2).

Ostensive Browsers. Several systems for exploratory information-seeking have helped users overcome difficulties in query formulation by attempting to infer the "ostensive relevance" of items [Cam96] directly from the user's interactions with the results. The ViGOR system [?] supplements video recommendation using information gained from user-created groupings of relevant results. Apolo, by Chau et al. [CKHF11], similarly allowed users to place results into groups and used belief propagation to suggest additional items of potential relevance. Comparing an osten- 

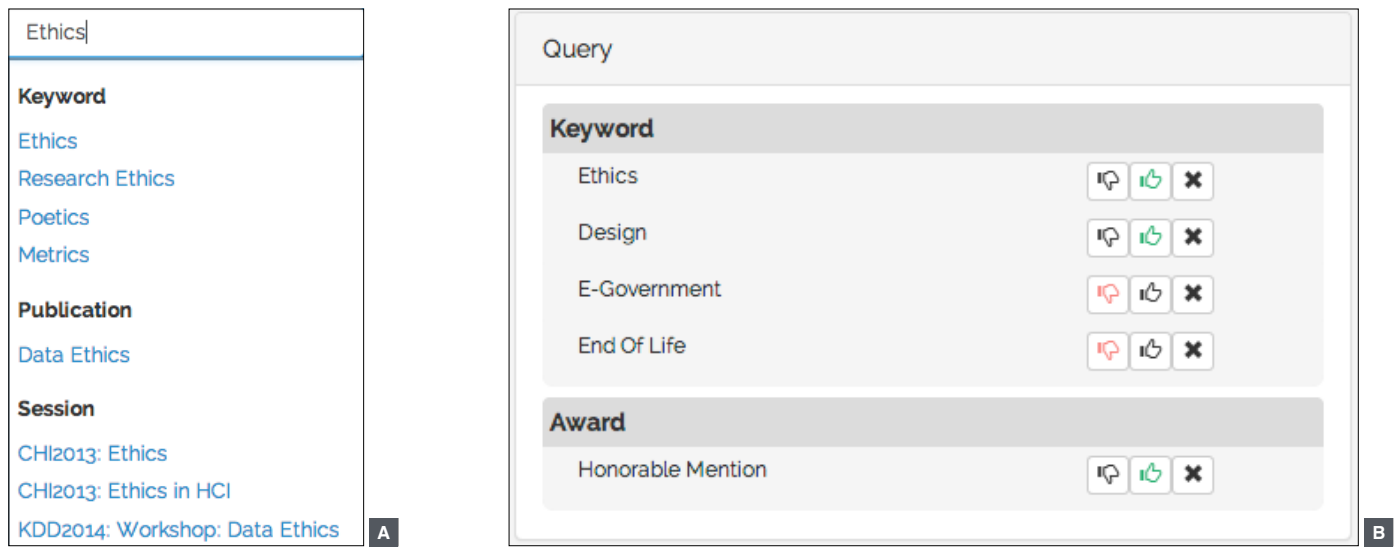

Figure 2: (a) Free-text search allows users to identify items in the dataset matching their interest. (b) Items which have been "upvoted" or "downvoted" into the query are grouped together for easy reference.

sive browser to a traditional keyword-search interface for image retrieval, Urban et al. [UJvR06] found that the ostensive browser stimulated significantly more ideas for alternate searches and led to fewer dead ends.

Apolo provides an example of how allowing users to point directly to items to communicate relevance provides a powerful and flexible means of specifying search intent (G2). This interactive loop, with output (results) serving in part as input (queries), allows the user and system to work together to determine relevance (G3). Placing these items in a network visualization, as Apolo does, provides cues about context and relationships, opening avenues for subsequent exploration, but Apolo only shows direct relationships between items of a single type, limiting opportunities for associative browsing (? G4),. It is not clear how belief propagation might work over heterogeneous networks ( $\neg$ G1). In the conference publication dataset used to evaluate Refinery, for instance, high-degree nodes such as PublicationType Paper or particular Conferences might propagate relevance to large numbers of unrelated items.

\section{Introducing Refinery}

Based on these design guidelines and insights from existing systems, we have created Refinery, a prototype system for supporting associative browsing over large, heterogeneous information networks. While the approach described generalizes to arbitrary heterogeneous networks, we illustrate the system's features and implementation using data from the Confer project [BKM14]. This dataset captures publication from 13 academic conferences between 2012 and 2014 in fields such as HCI, Data Mining, and AI.

\subsection{Refinery in Use}

We illustrate Refinery's features and how the system can be used for associative finding and browsing through the fol- lowing example: Mae recalls an interesting talk she attended at a recent conference related to ethics in Human-Computer Interaction research. She can't recall the title or authors, but she does remember that the paper won an Honorable Mention. She would like to find that paper, as well as related papers which may be of interest.

Free-Text Search. Users exploring with Refinery are presented initially with a single search box, inviting them to enter a free-text query. Mae might start by entering "ethics." The search box matches her text against labels of entities in the network, pulling up the relevant matches shown in Figure 2(a). Mae selects the Keyword Ethics. She similarly uses the free-text box to find an additional query item based on what she already knows, adding Award Honorable Mention.

Sidebar. Once an initial keyword has been selected, the main Refinery interface appears. The Sidebar groups the items which comprise the query at the top for quick reference. Associated items are suggested below in the "facets" panel. These items are grouped by type and sorted within type by relevance score. Browsing Keywords, Mae uses the thumbs-up button to "upvote" Keyword Design, shifting focus towards this term. She sees the Keywords End of Life and E-Government and recalls that the paper she seeks doesn't address these issues, so she "downvotes" these using the thumbs-down button to shift focus away from these topics. Each item she selects updates her query and the suggested facets being shown. Figure 2(b) shows how the query panel represents the current state of her query.

Graph View. Figure 3 shows the Graph View for this query, which shows the most relevant items returned by the system along with their connections. Here, Mae can browse through items by mousing over each one to see connections highlighted, as illustrated earlier in Figure 1. By clicking on an item, she can see data stored along with that item. She clicks on a Publication Categorised Ethical 
S. Kairam et al. / Refinery: Visual Exploration of Large, Heterogeneous Networks through Associative Browsing

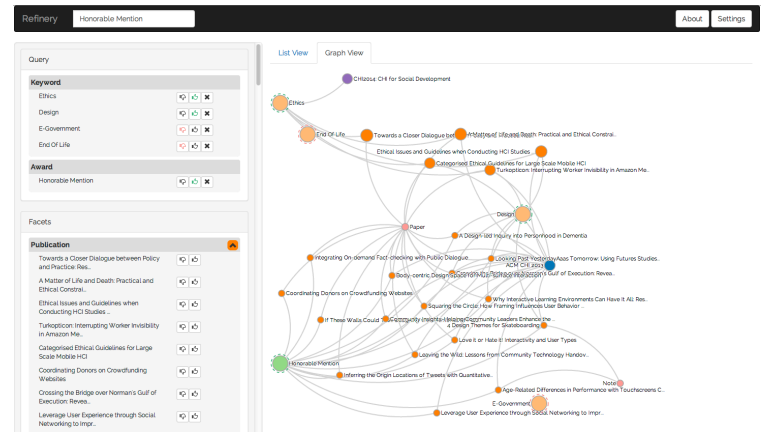

Figure 3: Refinery's Graph View clearly displays items most relevant to the query along with their relationships.

Guidelines for Large Scale Mobile HCI and reads the abstract. This isn't the paper she is looking for, but it is very similar, so she adds it to her query in order to attract related content. Doing so causes the Session CHI2013: Ethics in HCI to appear, which she also adds to the query.

List View. At this point, Mae feels that she is pretty close to finding the paper that she wanted. She switches over to the List View (shown in Figure 4), which shows all the items returned for her query in a single list, ranked by overall relevance score. By clicking the headers for facet groups in the Sidebar, she hides all types except for Publications, allowing her to easily scroll through a ranked list of relevant Publications. The third item in the list is the paper she had hoped to find, Publication Benevolent Deception in $\mathrm{Hu}-$ man Computer Interaction. Because entries in the List View provide a more complete view of items, she observes that this paper didn't use her original keyword, despite being on a closely related topic. She scrolls through this list, finding several papers which are closely related to this paper in various ways.

\subsection{Modeling the Data}

Refinery internally represents information collections as a heterogeneous association network. Node and edge types for the Confer dataset are given in Tables 2 and 3. Undirected relationships are represented using reciprocal directed edges. Each type of edge has a weight attribute, assigned initially based on intuitions about the data (e.g. Publication-Author edges represent stronger relationships than $P u b$ lication-Conference edges) and adjusted through empirical tuning. Our experience with the Confer dataset found that search results were not highly sensitive to exact choices of edge weight. In the discussion section, we consider how edge weights might be learned or refined based on user interaction with the system.

Each node has unique key and label attributes used for indexing and display, but nodes and edges are otherwise schema-less, with arbitrary attributes. This representation al-

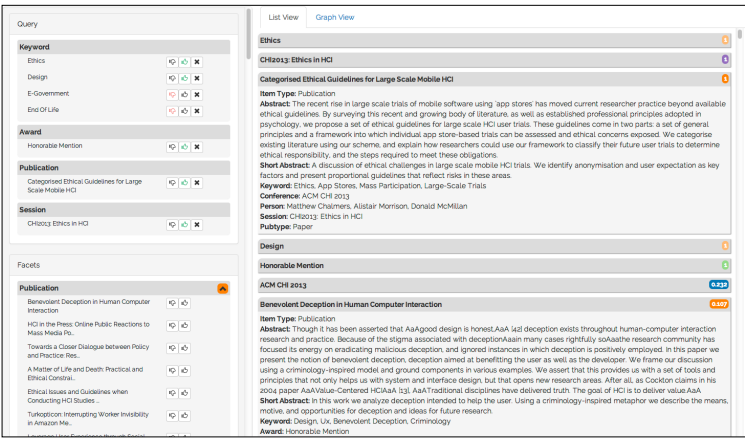

Figure 4: Refinery's List View ranks items by overall relevance. Users can easily hide or show particular types using toggles in the Sidebar.

lows us to represent diverse types of information, including non-textual or multimedia content, and their relationships in a common data structure for browsing and ranking (G1).

Choosing an ontology for nodes and edges is an important decision potentially affecting system performance and utility. For instance, rather than separating Publication-Type as its own node type, we could have simply made this an attribute of Publication nodes. As a heuristic, we separated all values for categorical attributes into individual nodes and treated any fields with values generally unique to specific nodes as attributes. Some decisions about how to model a particular dataset may come down to subjective preference or empirical tuning, and a system for projecting tabular data into different network representations, such as Ploceus [?], could prove useful for this task.

\subsection{Searching the Association Network}

Refinery's interface allows users to specify queries directly using collections of nodes in the network, helping to achieve (G2). At a high level, Refinery identifies nodes relevant to the query set by searching the association network through simulated random walks, an approach sharing properties with PageRank [BP98] and Random Walk with Restart (RWR) [TFP06]. Scores are computed for individual query nodes and then combined to compute relevance scores for each node in relation to the entire query set.

For each individual query node, the system simulates multiple random walks starting at this node following outgoing edges. At each step, the walker at some node $i$ will either (1) choose an edge $e_{i \rightarrow j}$ from the set of edges $e_{i}$ outgoing from $i$ with probability proportional to the weight of the edge, or (2) stop with some "halting" probability $p_{H}$. The probability that the walker transitions from a node $i$ to a connected node $j$ is given by:

$$
p\left(e_{i \rightarrow j}\right)=\frac{w\left(e_{i \rightarrow j}\right)}{p_{H}+\left(1-p_{H}\right) \sum_{e \in e_{i}} w(e)}
$$


S. Kairam et al. / Refinery: Visual Exploration of Large, Heterogeneous Networks through Associative Browsing

\begin{tabular}{|l|r|}
\hline Node Type & Count \\
\hline \hline Conference & 13 \\
\hline Session & 944 \\
\hline Persona & 48 \\
\hline Publication & 3200 \\
\hline Keyword & 4465 \\
\hline Publication Type & 25 \\
\hline Award & 2 \\
\hline Person & 8203 \\
\hline Affiliation & 1822 \\
\hline Total & $\mathbf{1 8 7 2 2}$ \\
\hline
\end{tabular}

Table 2: Node types and counts in the study dataset.

High values of $p_{H}$ produce shorter walks, leading to more conservative, locally-relevant results. Low values of $p_{H}$ produce globally relevant results, similar to those given by PageRank. For each node $i$ in the query set, the score for each other node $j$ in the network is given by the frequency with which simulated walks originating at $i$ ended at $j$. We assign a score of $n_{i}=0$ for any node that is never the destination for a walk from $i$.

For queries composed of multiple nodes, we combine results through simple addition or subtraction of scores. If the user selects two nodes $i$ and $j$, we simply add the scores $n_{i}+n_{j}$, and rank them accordingly. This approach is intuitive and captures content which is strongly associated with either query item and content which may be moderately associated with both. If, instead, the user had "upvoted" $i$ and "downvoted" $j$, we would subtract the scores $n_{i}-n_{j}$. In this case, an item associated with $j$ might still appear highly ranked if its association to $i$ is strong enough.

We choose this approach for a few reasons related to our design guidelines, especially (G1). First, it allows us to rank textual and non-textual data of any type, in relation to query nodes of any type. The algorithm doesn't require committing to or pre-computing an a priori interest function. In addition, we aren't committed to computing a separate notion of user interest, such as query-document relevance, for each type of node, which is especially useful when we consider non-textual nodes.

By simulating random walks at query time rather than pre-computing scores, we can easily handle dynamically updated data, such as social media or event logs. In addition, parameters such as edge weights or halting probability could potentially be tuned by users on-the-fly during search sessions to manipulate results. This approach satisfies our requirements, is suitable for any type of data which can be modeled as a heterogeneous network, and performs as expected for the dataset outlined here. In the discussion, we present some additional datasets to which we have successfully applied this approach.

\begin{tabular}{|l|r|r|}
\hline Edge Type & Weight & Count \\
\hline \hline Publication - Author & 10 & 11252 \\
\hline Publication - Session & 5 & 2780 \\
\hline Publication - Keyword & 5 & 7174 \\
\hline Session - Chair & 4 & 46 \\
\hline Publication - Conference & 3 & 3182 \\
\hline Session - Person & 3 & 225 \\
\hline Publication - Award & 2 & 134 \\
\hline Conference - Session & 2 & 944 \\
\hline Session - Keyword & 2 & 2864 \\
\hline Person - Affiliation & 2 & 6130 \\
\hline Publication - Pub Type & 1 & 1604 \\
\hline Total & - & $\mathbf{7 2 6 7 0}$ \\
\hline
\end{tabular}

Table 3: Edge types and counts in the study dataset.

\subsection{Interaction and Visual Representation}

The primary interaction method in Refinery is adding and removing nodes to and from the query using the "upvote" and "down vote" buttons. This approach, successfully used to foster continuous exploration in systems such as $\mathrm{Mr}$. Taggy [KNPC09], makes the process of providing relevance feedback to refine search intent simple and lightweight. When users update the query set, results update instantly to better reflect the inferred search intent. By enabling a quick interactive loop, we engage users in a continuous, uninterrupted dialog with the system (G4).

Refinery provides multiple views on results, offering multiple contexts for retrieving information. Highly ranked items are shown grouped by type in the Sidebar, ordered by score in the List View, and clustered with associated nodes in the Graph View. In the List and Graph Views, higherscoring nodes are shown with more contextual information than lower-scoring nodes, concentrating the user's attention on items more likely to lead to recognition of desirable content or serendipitous discovery.

Because our intent was to foster a novel style of exploration, we endeavored to maintain familiar graphical representations, such as the force-directed node-link diagram, to avoid overwhelming new users. In the following subsection, we describe the process which led us to the current design, and in the discussion we consider other potential options for visually presenting results.

\subsection{System Development}

The current version of Refinery was the result of two prior iterations.

An initial pilot with 12 users compared a force-directed layout to a more constrained layout with nodes grouped radially around a circle. The radial layout grouped nodes by type, but the force-directed layout promoted perception of clusters of related nodes of mixed types (better supporting 
G4). Participant feedback indicated that the force-directed layout was 'simpler' to understand.

In the second iteration, we deployed a version publicly for two weeks around the CHI 2014 conference. Conference attendees were invited to use the system to find talks and people of interest. This version contained only the Graph View (no List View). This deployment attracted over 400 unique users, and roughly half of these conducted some exploration. We observed users building diverse query item sets, validating our intuitions that this would be an expressive means of formulating queries. Requests for more browsable lists of items led to our incorporation of the complementary List View, significantly speeding up result browsing.

Refinery's query engine is implemented in Python, using the network library to store and search the association graph. The interface is implemented in HTML/CSS/JS, using the jQuery and $\mathrm{d} 3$ [BOH11] libraries for interaction and visualization. $d 3$ 's force-directed layout is used for the Graph View. As discussed earlier, the system makes available several tunable parameters; however, we chose not to expose these to users in the study. We ran 2,000 walk iterations for each query node, with $p_{H}=0.4$ and edge weights as described above.

\section{User Study}

In order to identify ways in which Refinery might achieve our goal of supporting associative browsing over heterogeneous networks, we conducted a user study with 12 participants. We specifically recruited academic researchers with expertise in areas covered by the data; we were interested in how Refinery might yield insights extending beyond those uncovered by existing tools, such as Google Scholar.

Participants. We recruited 12 academic and industry researchers (7 Female / 5 Male, Age: $\mu=27.4, \sigma=3.3$ ); all had at least some graduate study in the areas of HCI, Information Visualization, or NLP. All participants reported moderate-to-expert familiarity with at least 3 conferences in our dataset. Each experiment session lasted approximately one hour; participants were offered $\$ 25$ in compensation.

Procedure. Sessions started with a brief introduction and explanation of the study procedure. Participants were given a walkthrough of the dataset and a pre-survey assessing familiarity with conferences in the data. The main study task asked participants to explore the data at their leisure for up to 15 minutes; they were informed that they could conclude sooner if they felt that their exploration had drawn to a close. At the end of the session, each participant completed a brief questionnaire about the system (adapted from Bernstein et al.'s evaluation of Eddi [?]) and engaged in a 10-minute interview about their experience.

During the exploration task, we followed a think-aloud protocol, asking subjects to describe their interactions with the system and data. We specifically asked that they report any interesting observations about the data, including errors, surprising patterns or connections, and confirmation or rejections of prior hypotheses or intuitions they may have had. All sessions were run using Google Chrome on a 15-inch MacBook Pro. Study sessions were captured using query logging, screen-capture, and audio-recording.

\section{Study Results}

We present some behavioral observations and feedback regarding the system in order to assess ways in which Refinery did or did not successfully match our initial design goals. We use codes P1 to P12 throughout when providing examples or quotes from individual participants.

System Usage. All participants used the system actively, including a substantial number of unique nodes as part of their queries over the course of a session (Unique query nodes per user: $\mu=12.5, \sigma=4.9$ ). Nodes were combined into diverse queries; participants created a large number of unique query combinations in each session (Unique query sets per users: $\mu=17.8, \sigma=7.2$ ). No participant expressed frustration over unintended queries, indicating that Refinery's design supports rapid and expressive query formulation (G2).

Most participants composed query sets composed of diverse node types (Unique types used per user: $\mu=3.67$, $\sigma=1.23$ ). As we believe Refinery is unique in allowing users to specify queries in terms of heterogeneous node sets, we were encouraged to see this feature used extensively. Every node type, except for Persona was used in at least one query by at least one participant. Another method of diversifying queries, "down voting" nodes, was used at least once by the majority (7/12) participants in our study.

Subjective Feedback. Based on verbal feedback and our observations of the exploration sessions, participants appeared to enjoy using the system. All (12/12) participants explored for the full 15 minutes, and several asked to continue exploring after the task period had concluded. By design, our study engaged subjects in exploring content information over which they already had some expertise. Despite this high level of familiarity, every (12/12) participant found novel items of interest, based on their own self-report.

One participant, for instance, explored topics relevant to her current research, finding several novel Publications which she noted down to review later on her own. Previously unaware of the specific design-related Keyword attached to these Publications. she had missed them earlier when searching using Google Scholar. When discussing how she found them using Refinery, she said, "It gave me suggestions for things I might not actually have searched for, but were quite related." [P3].

Another participant searched for ideas to help plan an upcoming research project; he was surprised to find work focused on algorithms within the HCI community. 


\begin{abstract}
It certainly made me more interested in the topic than I was before...I didn't have a genuine deeplyseated interest in it. Now, I think I genuinely do, if only because I see that the way that it is interesting to $\mathrm{HCI}$ is the applications of it, and the people whose work...interested me, intrinsically, relates to algorithms and applications thereof. [P12]
\end{abstract}

In this case, the user's perspective on his own research was changed by the 'serendipitous' experience of encountering novel information along with associations to content which was familiar and meaningful to him.

Questionnaire Responses. In a post-study questionnaire, participants indicated their level of agreement with several statements using a 7-point Likert scale, from 1 (Strongly Disagree) to 7 (Strongly Agree). Participants rated the system highly in terms of interestingness $(\mu=6.33, \sigma=0.89$ ), enjoyability $(\mu=5.33, \sigma=1.30)$, and flexibility $(\mu=5.25$, $\sigma=1.36$ ). Participants disagreed quite a bit about the extent to which they found the system "overwhelming" $(\mu=3.08$, $\sigma=2.02$ ), pointing to possible individual differences in preferences for associative browsing.

\subsection{Browsing Strategies.}

The study also offered the opportunity to use Refinery as a probe for studying users engaged in exploratory information-seeking within a realistic, but controlled environment. We observed several common low-level strategies which participants mixed and matched while browsing.

Every (12/12) participant engaged at least once in refining (Refinery's principal feature), iteratively adding items to the query set in order to focus exploration within a subarea of the data. After building up a query set through refining, the majority of participants (10/12) shifted the focus of their query by traversing, removing initial query items until they had navigated to a novel area in the data.

Instead of traversing to a new location, participants sometimes retreated (5/12), removing newly added items from the query set to return to an earlier view of the data. In half of the sessions (6/12), we observed at least one situation in which one or more query items served the function of bridging between two areas. Adding the "bridge" item(s) prompted participants to remove all other existing query items, add new query items, and continue exploring a new, but related, area of the data. While identifying these strategies was not the focus of the present study, they provide an interesting means of summarizing browsing behavior and could potentially serve as the subjects for future research.

\subsection{Study Limitations}

Our study was open-ended by design, as we aimed to observe users browsing in a realistic and unconstrained setting.
This study design, however, did not allow for a quantitative comparison of browsing performance against existing tools, something we would like to achieve in the future. Participants frequently compared Refinery to existing academic search tools backed by more extensive corpora (allowing access to content from before 2012). In our ongoing research, it would be helpful to observe users browsing more complete datasets to avoid disappointment based on missing content.

In addition, as the interface included several novel elements, some participants required several minutes before feeling that they had 'gotten the hang of it.' The current implementation of Refinery does not preserve node positioning in the Graph View across transition; adopting techniques for doing so may aid users in tracking objects across multiple queries. Despite our efforts to create a realistic task environment, observing participants familiar with the system browsing in self-prompted scenarios may yield different results.

\section{Discussion \& Future Work}

We are encouraged by participants' positive comments, as well as the underlying reasons, which closely aligned with our proposed design guidelines. Several participants explicitly called out the distinction between the associative style of browsing enabled by Refinery and that afforded by more traditional retrieval interfaces, such as Google Scholar.

Google Scholar is great when you know what you're looking for...once you know what you're looking for, it's very easy to recognize. But it's not so easy to find things which are ill-defined. [P12]

It wasn't like when you go to Google, and you know exactly what you're searching for, and you just find it. This is more like I'm trying to explore this space and it's a really wide range of things. So being able to put poles where things would gather around the ideas that were really interesting to me was really awesome, and I found articles that I wouldn't have looked at. [P3]

One participant described how interaction with the data aided her in focusing in exploration, clearly illustrating some of our goals in designing for associative browsing.

You don't know what questions you're going to have if you don't know what the layout is...that's how you develop good questions. [P4]

An important area of future work is exploring how alternative modeling decisions for the association network will impact result quality and exploration strategies. For different types of data, we might consider adding nodes based on computed connections. For text corpora, for instance, we might compute latent topics using LDA [BNJ03] and add nodes for these, connecting documents with similar semantics. For image corpora, we could similarly create nodes to represent computed image characteristics. 

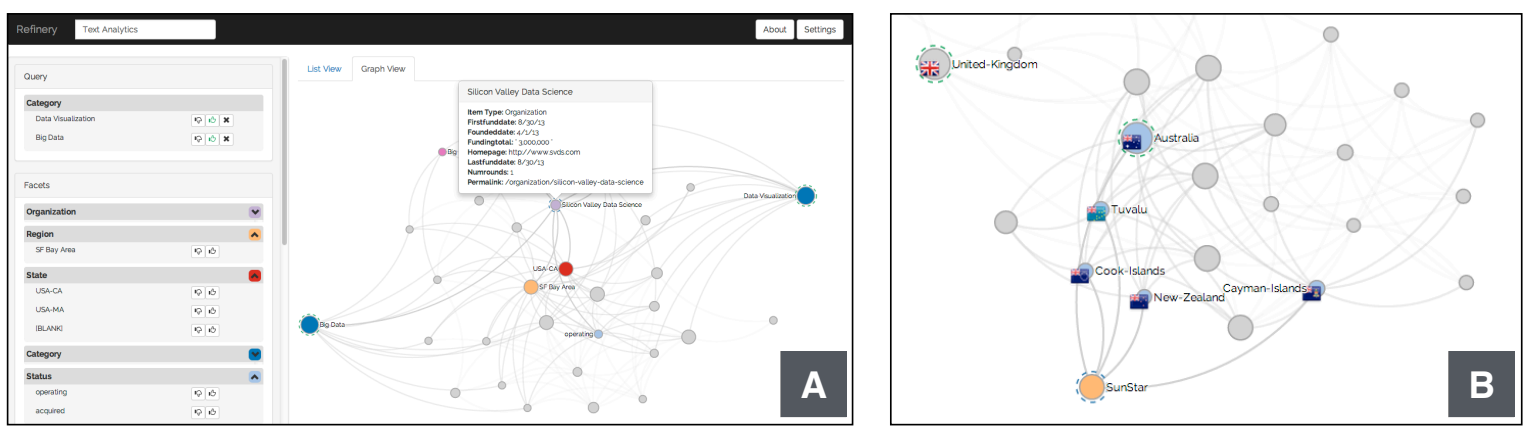

Figure 5: (a) Exploring San Francisco Bay Area startup company data from Crunchbase. Looking for companies related to Big Data and Data Visualization, the user has identified one which may be of interest. (b) Exploring national flags by querying for the UK and Australia, the user highlights a node representing presence of sun or star imagery, highlighting a graphical element shared by several Commonwealth nations.

While our search algorithm performed admirably for the Confer data, future work might compare it against related alternatives. One obvious limitation of the current algorithm is that walks from a query node never reach items outside of the node's weakly connected component. We could potentially address this problem by introducing random jumps, as in PageRank [BP98], for instance. The approach of simulating random walks, as we suggest here, also opens up possibilities for exploring parallelization as a means for speeding up result computation and scaling to larger datasets.

Our participants expressed enthusiasm for similar support for exploring other types of collections using a similar browsing style. In Figure 5(a), we illustrate how Refinery might be used to explore data about San Francisco Bay Area startup companies from Crunchbase [Cru14]. Here, the user is browsing companies related to Big Data and Data Visualization, discovering a recently-formed company which shares both tags and mousing over it for more information.

Figure 5(b) illustrates browsing a dataset of national flags [BL13] which combines textual and non-textual data. Here, flags are linked to nodes representing common graphical elements (e.g. crosses, saltires, or animate figures) and demographic features (e.g. language, continent, religion). Here, after querying for the United Kingdom and Australia, the user mouses over a node representing sun and star imagery, highlighting a graphical feature common to the flags of several Commonwealth countries. We note here that the flag nodes are simply images, retrieved using only the network structure, illustrating how our approach can adapt flexibly to multimedia content. These nodes could just as easily represent audio files or video files without the need to compute content-specific user interest functions. While our ranking approach generalizes easily to such multimedia data, we might consider alternate visual presentation approaches to aid in navigating such collection.

Media collections, product databases, and news repositories are just some of the areas ripe for next-generation visual interfaces to support associative browsing. We are eager to continue iterating on all of these aspects of Refinery in order to solve important problems related to exploratory information-seeking in these various areas.

\section{Conclusion}

We have introduced Refinery, an interactive visualization system designed to support bottom-up exploration of large, heterogeneous networks through associative browsing. Refinery's design was informed by guidelines and techniques derived from examination of prior analysis of and existing systems built for exploratory information-seeking.

Our approach incorporates a novel application of randomwalk based algorithms to the domain of degree-of-interest visualization of heterogeneous networks. The user interface allows users to specify the 'frontier' of their knowledge using collections of nodes of various types and visualizes results to effectively displays context and connections which spur insights and further exploration.

Feedback from a user study with 12 researchers browsing publication data from familiar research areas demonstrated that Refinery is effective in aiding exploratory informationseeking, even in cases where users had high levels of expertise in the knowledge domain explored. We hope that the guidelines and strategies considered here are informative for future visualization systems intending to support associative browsing as a strategy for exploration.

\section{References}

[AP84] Anderson J. R., Pirolli P. L.: Spread of Activation. Journal of Experimental Psychology 10, 4 (1984), 791-798. 2

[ASTd09] André P., Schraefel M., Teevan J., Dumais S. T.: Discovery Is Never by Chance : Designing for ( Un ) Serendipity. In Proc. ACM C\&C (2009), pp. 305-314. 2, 3

[Bat89] BATES M. J.: The design of browsing and berrypicking techniques for the online search interface. Online Review 13 (1989), 407-424. 2

[Be193] BELKIN N. J.: Interaction with Texts : Information Retrieval as Information-Seeking Behavior. In Proc. of Info. Retr. (1993), pp. 55-66. 1, 2, 3 
[BKM14] Bhardwaj A., Karger D., Madden S.: Confer Project, 2014. URL: http://confer.csail.mit.edu/. 4

[BL13] BACHE K., LICHMAN M.: UCI Machine Learning Repository, 2013. URL: http://archive.ics.uci. edu/ml. 9

[BNJ03] Blei D. M., Ng A. Y., JoRdan M. I.: Latent Dirichlet Allocation. Journal of Machine Learning Research 3 (2003), 993-1022. 8

[BOH11] Bostock M., Ogievetsky V., Heer J.: D3: DataDriven Documents. IEEE transactions on visualization and computer graphics 17, 12 (2011), 2301-9. 7

[BP98] BRIN S., PAGE L.: The anatomy of a large-scale hypertextual Web search engine. Computer Networks and ISDN Systems 30 (1998), 107-117. 5, 9

[Bru93] BRUZA P. D.: Stratified Information Disclosure: A Synthesis between Information Retrieval and Hypermedia. $\mathrm{PhD}$ thesis, University of Nijmegen, The Netherlands, 1993. 2, 3

[Cam96] CAMPBELL I.: The ostensive model of developing information needs. PhD thesis, University of Glasgow, Scotland, 1996. 3

[CKHF11] Chau D. H., Kittur A., Hong J. I., Faloutsos C.: Apolo : Making Sense of Large Network Data by Combining Rich User Interaction and Machine Learning. In Proc. ACM CHI (2011), pp. 167-176. 3

[CMF08] Chau D. H., Myers B., Faulring A.: What to do when search fails: finding information by association. Proc. ACM CHI (2008), 999-1008. 1

[CNO2] CARD S. K., NATION D.: Degree-of-Interest Trees: A Component of an Attention-Reactive Interface. In Proc.ACM AVI (2002). 3

[Cru14] Crunchbase, $2014 . \quad$ URL: http://www. crunchbase.com/. 9

[DCW11] Dörk M., Carpendale S., Williamson C.: The Information Flaneur : A Fresh Look at Information Seeking. In Proc. ACM CHI (2011), pp. 1215-1224. 1, 2, 3

[FLGD87] Furnas G. W., Landauer T. K., Gomez L. M., DUMAIS S. T.: The Vocabulary Problem in Human-System Communication. CACM 30, 11 (1987), 964-971. 1, 2, 3

[Fur86] Furnas G. W.: Generalized fisheye views. ACM SIGCHI Bulletin 17, 4 (1986), 16-23. 3

[HC04] HEER J. M., CARD S. K.: DOITrees Revisisted: Scalable Space-Constrained Visualization of Hierarchical Data. In Proc. ACM AVI (2004), pp. 421-424. 3

[KÖ5] KÄKI M.: Findex: search result categories help users when document ranking fails. Proc. ACM CHI (2005), 131-140. 3

[KNPC09] KAmmerer Y., NAIRn R., Pirolli P., Chi E. H.: Signpost from the Masses : Learning Effects in an Exploratory Social Tag Search Browser. In Proc. ACM CHI (2009), pp. 625634. $2,3,6$

[LPP*] Lee B., Parr C. S., Plaisant C., Bederson B. B., Veksler V. D., Gray W. D., Kotfila C.: TreePlus: Interactive Exploration of Networks with Enhanced Tree Layouts, journal=IEEE TVCG, volume $=12$, number $=6$, pages $=1414$ 1426, year=2006. 3

[Mar04] MARCHIONINI G.: From information retrieval to information interaction. In Advances in information retrieval, McDonald S., Tait J., (Eds.). Springer Berlin Heidelberg, 2004, ch. From Infom, pp. 1-11. 2, 3
[Mar06] MARCHIONINI G.: Exploratory search: From finding to understanding. CACM 49, 4 (2006), 41-46. 3

[MS88] MARCHIONINi G., SHNEIDERMAN B.: Finding facts vs. browsing knowledge in hypertext systems. Computer 21, 1 (Jan. 1988), 70-80. 1, 2

[OJ93] O'DAY V. L., JEFFRIES R.: Orienteering in an Information Landscape: How Information Seekers Get From Here to There. In Proc. ACM CHI (1993), pp. 438-445. 1, 2

[RBSW01] Rodden K., Basalaj W., Sinclair D., Wood K.: Does Organisation by Similarity Assist Image Browsing? In Proc. ACM CHI (2001), pp. 190-197. 3

[TAAK04] TeEvan J., Alvarado C., Ackerman M. S., Karger D. R.: The Perfect Search Engine Is Not Enough : A Study of Orienteering Behavior in Directed Search. In Proc. ACM CHI (2004), vol. 6, pp. 415-422. 1, 2, 3

[TFP06] Tong H., Faloutsos C., PAn J.: Fast Random Walk with Restart and Its Applications. In Proc. ICDM (2006), pp. 613-622. 5

[tHPvdW96] Ter Hofstede A., Proper H., VAN Der Weide T.: Query formulation as an information retrieval problem. The Computer Journal 39, 4 (September 1996), 255-274. 1, 2, 3

[TT73] Tulving E., Thomson D. M.: Encoding Specificity and Retrieval Processes in Episodic Memory. Psychological Review 80, 5 (1973), 352-373. 2, 3

[UJvR06] Urban J., Jose J. M., VAn RiJsbergen C.: An Adaptive Approach Towards Content-Based Image Retrieval. Multimedia Tools and Applications 31, 1 (2006), 1-28. 4

[vHP09] VAN Ham F., PERER A.: "Search, Show Context, Expand on Demand": Supporting Large Graph Exploration with Degree-of-Interest. IEEE TVCG 15, 6 (2009), 953-960. 3

[WKDS06] White R., Kules B., Drucker S. M., SchraeFEL M.: Supporting Exploratory Search. CACM 49, 4 (2006), 36-39.

[YSLH03] YEE K.-P., Swearingen K., Li K., Hearst M. A.: Faceted metadata for image search and browsing. In Proc. ACM CHI (New York, New York, USA, 2003), ACM Press, p. 401.3 